\title{
A Photoelectric Recording Interferometer for Measurement of Dimensional Changes
}

\author{
Richard N. Work
}

\begin{abstract}
A method is given for the photoelectric recording of light intensity at a reference point in an image of an interference pattern for the automatic plotting of fringe count versus thermocouple electromotive force in interferometric dilatometry. Application of the technique to the measurement of coefficients of expansion and to the determination of transition temperatures in rubber-like materials over a temperature range of $-185^{\circ}$ to $+185^{\circ} \mathrm{C}$ is given. Transitions can be located to a precision of \pm 0.5 degree $\mathrm{C}$ and coefficients of expansion found to a precision of \pm 5 percent.
\end{abstract}

\section{Introduction}

Many investigations require a study of the changes in dimension of a substance that take place upon a change in temperature. The temperatures at which phase transitions or second-order transitions occur may be located from such data, and the values of the expansivities at various temperatures may be found.

Crystallization in rubber, for instance, has been studied by means of X-ray diffraction, birefringence, specific heat, interferometric, and volume dilatometric methods. The second-order transition of rubber has been studied by volume dilatometry, interferometry, specific heat, and index of refraction measurements.

The study of transition phenomena by the interferometric method requires no confining liquid, may be adapted to wide temperature ranges, and is semiautomatic in operation. The application is limited only to materials that do not flow under small stress.

This paper deals with an improved interferometric method of determining the relationship between length and temperature of solid materials. Novel features of the present work include the photoelectric counting of fringes, the automatic plotting of fringe count against thermocouple emf, and the easy adjustment of the fringe pattern. Illustrative data obtained from measurements on rubber are given, but the application of the method described is not restricted to this type of material.

Merritt [1] ${ }^{2}$ and Saunders [2] have described in detail the interferometric apparatus and procedure. The application of the method to measurements on rubber-like materials has been described by Wood, Bekkedahl, and Peters [3]. The method may be described very briefly as follows. If the separation of two optically flat, transparent plates is determined by the thickness of three spacers, a few millimeters thick but equal in thickness to within a few wavelengths of light, and if these plates are illuminated with parallel monochromatic light normal to the plane of their faces, a pattern of interference fringes will be observed. If, now, the thicknesses of the specimens are caused to change by a variation in

The work reported here was supported by the Office of Naval Research and the Office of the Quartermaster General as part of Project ONR (QMC) NR$033-314$.

${ }^{2}$ Figures in brackets indicate the literature references at the end of this paper. temperature, the separation of the plates will be altered, and there will be a corresponding variation in the fringe pattern evidenced by a motion of the fringes. The change in separation of the plates at a given point is related to the motion of the fringes by the following relation

$$
\Delta l=\frac{\Delta N \lambda}{2},
$$

where $\Delta l$ is the change in separation; $\Delta N$ is the number of fringes passing the point of reference; and $\lambda$ is the wavelength of light used. By the use of a suitable illuminating and viewing instrument, the motions of the fringe pattern can be measured quantitatively, and the change in dimension associated with a given temperature interval can be obtained from eq 1 .

The visual interferometric method requires continuous and undivided attention, which may account for the fact that its adoption has been limited. Various photographic methods of recording the changes in the fringe pattern have been developed $[4,5,6]$ to eliminate this objection. A photoelectric method of recording the fringe count shows certain advantages over the photographic methods, including the elimination of film-processing and the presentation of data on a strip chart in a form suitable for immediate determination of transition temperatures and estimates of expansivities. The quantitative determination of the coefficients of expansion requires less processing than other interferometric methods. The course of a measurement can be followed and the procedure modified when it is seen to be necessary. The speed to this photoelectric method can be made sufficiently high to be able to count the rapidly moving fringes during rapid change in volume that accompany phase transitions.

The photoelectric method utilizes the changes in light intensity at a reference point in the fringe pattern as the fringes move past this point. These changes are detected by a photomultiplier tube, and the amplified output of the tube is automatically plotted against time on a strip-chart recorder. A switch, actuated by the recorder movement, produces a current pulse upon the passage of each 


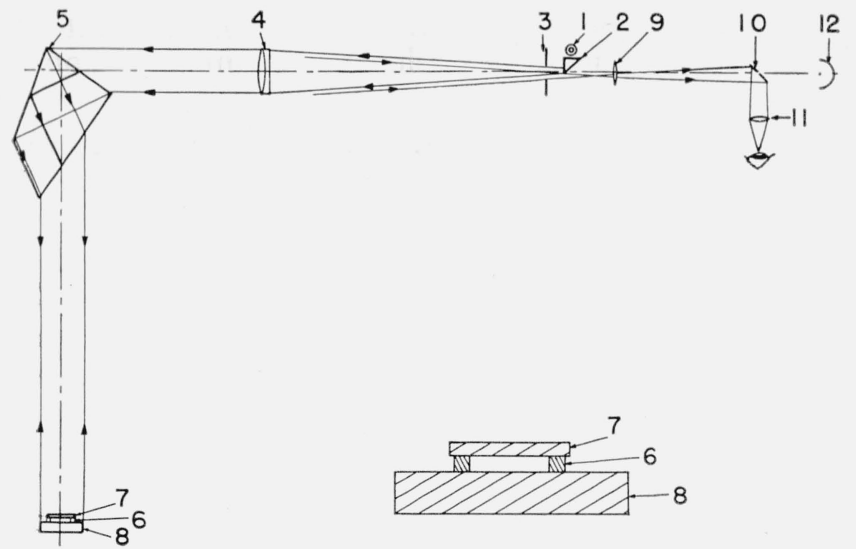

Figure 1. Optics of the interferometric illuminating-viewing instrument.

Sce text for explanation of numbered items. Enlarged section of 6, 7, and 8 is shown at the right.

fringe. Simultaneously, the temperature of the specimen, as indicated by the emf of a thermocouple embedded in a piece of material of similar size to the specimen, is plotted against fringe count on a second stripchart recorder, which has been so modified that the current pulse from the time-intensity recorder actuates a mechanism that moves the chart a unit distance for each fringe.

\section{Apparatus}

\subsection{Interferometer}

A Pulfrich type [7] viewing instrument, similar to that described by Merritt and modified according to figure 1 forms the optical part of the apparatus used here. The light source is a helium tube, 1, located above a small right-angle reflecting prism, 2 , which places the effective source very near to the optic axis of the instrument. After traversing an iris diaphragm, 3, which limits the effective size of the source, the rays are rendered parallel by the lens, 4. The light is deviated through approximately $90^{\circ}$ and dispersed in passing through the Pellin-Broca prism, 5. By rotating this prism about an axis perpendicular to the plane of the paper, light of a wavelength corresponding to any one of the spectral lines in the source may be made to fall perpendicularly upon the interferometer plates, separated by three samples, 6 . The rays reflected from the bottom surface of the top plate, 7 , and from the top surface of the bottom plate, 8 , interfere in a manner determined by the separation of the plates and form a pattern of interference fringes. The two surfaces of each interferometer plate are inclined at an angle of 20 minutes to eliminate unwanted reflections from the upper surface of the top plate and the lower surface of the bottom plate. The reflected light returns through the prism and collimating lens to form an image of the effective source in the lower half of the iris diaphragm, through which the light passes to the photoelectric detector. Images corresponding to other wavelengths present in the source fall above or below the opening and are stopped. An image of the interferometer plates is formed by lens, 9, and falls upon a mirror, 10, inclined at an angle of $45^{\circ}$ to the beam. This mirror, which contains a small aperture about $0.1 \mathrm{~mm}$ in diameter, permits inspection of the image through the side tube with the aid of lens, 11. Light passing through the aperture, which is about one-fiftieth the diameter of the image, falls upon the photosensitive surface of the photo-multiplier tube, 12.

Of the various spectral lines present in the light from the helium discharge tube, the yellow line, $5876 \mathrm{~A}$, is used because of its good visibility and because it shows the highest sensitivity-intensity product for the phototube used.

\subsection{Specimen Holder}

The specimen holder, figure 2 , is designed for use with rubber and rubber-like materials to be measured over a temperature range from $-185^{\circ}$ to $+185^{\circ} \mathrm{C}$. The discussion of the apparatus is divided for convenience into two sections, optical and thermal.

\section{a. Optical}

For this experiment, a useful fringe pattern is obtained when the surfaces of the two transparent plates are flat within two to three wavelengths and parallel within 20 wavelengths. If these surfaces are not parallel within this limit, 40 or more fringes will be seen to traverse the 2-cm-diameter plates making visual observations difficult. A more serious effect lies in the fact that the aperture in the inclined mirror becomes large compared to the width of each fringe. The variation in transmitted light upon the passage of a fringe then becomes too small to be detected photoelectrically.

If the material under test is hard, say a metal or ceramic, a specimen may be ground to one of the forms recommended by Saunders [2], making three connected, supporting structures the same length within a few wavelengths. However, there is considerable difficulty in cutting or molding three specimens of a soft rubber-like material to the same thickness within the required tolerance. Accordingly, the specimen holder has been designed so that the three specimens are supported by fused-quartz wedges, which in turn rest upon the bottom interferometer plate. Then, if the specimens are fixed laterally while the wedges are movable along a radius, a vertical adjustment of the top plate is possible. This motion enables one to bring the top plate into parallelism with the bottom plate without having to cut the specimens to close tolerances.

The wedges are $5 \mathrm{~mm}$ in width and $15 \mathrm{~mm}$ in length and vary in thickness from 2.00 to $2.15 \mathrm{~mm}$. The maximum travel of the wedge is of the order of $5 \mathrm{~mm}$, allowing a maximum vertical adjustment of $0.05 \mathrm{~mm}$. In practice, the maximum adjustment is used only for material that flows or creeps under the weight of the top plate, in which case the finding of an acceptable fringe pattern is fortuitous. Usually, however, vulcanized rubber samples and many unvulcanized materials can be easily cut or molded 

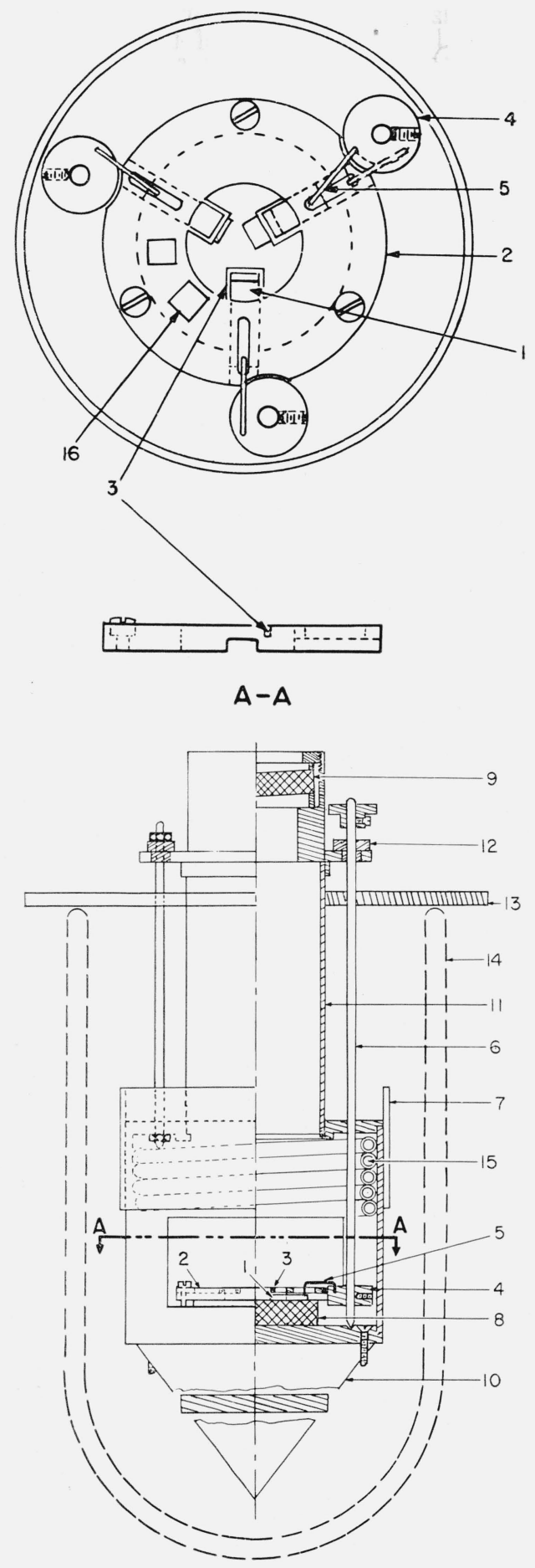

Figure 2. Sample holder.

See text for explanation of numbered items. Enlarged section of A-A is shown above.

to within $0.02 \mathrm{~mm}$. The uncertainty in thickness is, therefore, less than 1 percent of the usual thickness of the specimens ( 2 to $5 \mathrm{~mm}$ ).

The details of the wedge-adjusting mechanism are shown in figure 2 . The wedges, 1 , may be moved in radial slots milled in the brass plate, 2 . The central portion of this brass plate has been cut out to allow light to reach the bottom interferometer plate. Square enclosures, or wells, $5 \mathrm{~mm}$ square, 3, are located above the inner ends of the wedges, which extend into the open portion in the center of the brass plate. The specimens lie upon the quartz wedges and in these wells, the walls of which restrain the motion of the specimens when the wedges are moved.

Motion of the wedges is achieved by a lever drive, 4 , which is coupled to the wedge by means of a wire link, 5. The shaft, 6, fastened to the lever extends to the top of the holder where a knob allows the operator to make adjustments when the specimen is inaccessible. The side openings of the holder are closed by means of sleeve, 7 .

A window, 9, forms a closure at the top of the specimen holder. To avoid the reduction in contrast of the image caused by reflections from the window surfaces, the window is tilted at a small angle to deflect the light out of view.

Fogging and frosting of the window at low temperatures are eliminated by passing a stream of dry air over it. Frosting of the interferometer plates is avoided by circulating dry air in the specimen chamber.

\section{b. Thermal}

The specimens are cooled by liquid nitrogen pumped from a reservoir by means of a pressure bulb into a Dewar, 14, surrounding the specimen holder. The rate of cooling is determined by the rate at which the liquid nitrogen is pumped into the Dewar. A triangular brass wedge, $10,3 / 8$ in. thick, 2 in. high, and 3 -in. base is attached to the bottom of the specimen holder and acts as a heat transfer path of variable length and cross section to make the rate of cooling more nearly uniform with variation in liquid level. Unless such a device is used, the temperature in the sample chamber drops slowly until the liquid nitrogen level reaches the bottom of the specimen holder, at which time the temperature falls rapidly, making temperature control difficult.

Heat transfer to the surroundings is reduced by the use of a Bakelite tube, 11, as the center section of the specimen holder. Bakelite washers, 12, between the brass tie rods and the flange on the top of the specimen holder and between the wedge adjusting rods and the flange, reduce heat transfer along those paths. Further, closure of the space between the Dewar, 14, and the sample holder is made by means of a piece of Masonite, 13, lined with a soft plush cloth to make good contact with the edge of the Dewar. The amount of condensation and frosting on the exposed metal surfaces is small even when the sample is held near liquid nitrogen temperatures for several hours.

The temperature of the sample may be increased by means of a current in a resistance element located in the bottom of the Dewar. The heater current is adjusted with a variable transformer to obtain a suitable rate of heating.

Dry air passes through a copper tube, 15 , a 5 -turn helix inside the sample chamber with which it makes 
good thermal contact. The air stream then flows into the sample chamber and stirs the air, insuring a uniform temperature in the chamber.

\subsection{Amplifier}

The fringe recording circuit consists of a 931Aphotomultiplier tube to convert light changes into electric signals, suitable amplifier stages, and a strip-chart recorder.

The power supply for the photomultiplier tube is controlled with a variable transformer, allowing a wide range of gain. It is usually operated at about $90 \mathrm{v} /$ dynode for optimum signal-to-noise ratio.

The variation in the phototube current is about $5 \times 10^{-9}$ amp for the passage from a light band to a dark band of an interference fringe when operated at the conditions noted above. Noise currents from various sources, namely, the helium tube, phototube, stray fields, etc., are of the same order of magnitude. Therefore, a tuned circuit is employed in the amplifier to prevent the over loading of the amplifier by noise voltages. Since the helium tube is excited by a high-voltage transformer connected to the 60 -cycle line, the light is modulated with a fundamental frequency of $120 \mathrm{c} / \mathrm{s}$. A single-stage triode preamplifier is followed by a two-stage amplifier with negative feedback over a 120-cycle parallel $T$ rejection network. This results in a sharp peak at 120 cycles in the gain-frequency characteristic of the amplifier. A reduction in noise due to stray coupling to the line and poor filtering of the power supply could be made by a choice of some other frequency than the line frequency for the excitation of the helium lamp. Such an expedient was found to be unnecessary. The tuned amplifier is followed by a phase inverter-detector stage, which converts a single ended alternating-current signal to a balanced direct-current output, whose level is proportional to the peak input-signal voltage. The detector stage is followed by a balanced direct-current amplifier with an adjustable time constant. A time constant of $1,2,4,6$, or 8 sec may be chosen by means of a selector switch. The relatively long time constants average any fluctuations (noise) in the output that are of higher frequency than 1 to $1 / 8 \mathrm{c} / \mathrm{s}$. The available range permits the operator to choose a time constant that mostly corresponds to the frequency of variation of the desired signal, and that is sufficiently short so the signal is not appreciably attenuated. Normally the temperature is varied at a rate between $1 / 2$ and $1 \mathrm{deg} / \mathrm{min}$, which corresponds roughly to a fringe per minute for rubber specimens 2 to $5 \mathrm{~mm}$ thick. In this case the 8 -sec time constant is used. However, if the sample undergoes rapid crystallization, the fringes may move at rates up to 10 or $20 / \mathrm{min}$, in which case the 8 -sec time constant would appreciably reduce the amplitude of the output. In this case, a shorter time constant is used.

The output of the amplifier actuates a strip-chart recorder yielding a plot of light intensity at the phototube aperture against time. The recorder mechanism is fitted with a switch that is closed once per fringe. The switch consists simply of a vertical wire, the end of which is engaged by a projection on the recorder movement so as to make a momentary connection with a fixed contact. Contact is made only for motions in one direction. The momentary closing of the switch operates a relay, which in turn operates the fringe-counting solenoid on the temperature recorder.

The temperature of the specimens is indicated by the emf of two copper-constantan thermocouples in series. The reference junctions are in an ice-water bath, whereas the other junctions are placed in specimens of the same or similar material and of similar size to those under test. These specimens are placed in the thermocouple wells, 16, figure 2. The emf is recorded continuously on a recording-potentiometer. The recorder scale was expanded to cover the temperature range $-185^{\circ}$ to $+185^{\circ} \mathrm{C}$ in three spans. A precision of $\pm 0.5 \mathrm{deg}$ at the low-temperature end and $\pm 0.2 \mathrm{deg}$ at the high end is attainable. The potentiometer-recorder was calibrated with a laboratory potentiometer, and calibration scales were made. Emf's corresponding to temperatures at $1 \mathrm{deg}$ intervals, the values of which were taken from the International Critical Tables, were impressed upon the input terminals of the recording-potentiometer. The potentiometer was allowed to come to balance at each value of emf and a mark made. The temperature corresponding to any point on the chart can then be found simply by aligning the scale on the chart and reading the temperature from the scale corresponding to the point on the chart.

The chart paper drive of the recorder was modified so that an impulse from the fringe counting solenoid would move the paper a constant distance. The Brown Electronik recorder, model 153X11, was found to be well suited for this modification of the chart mechanism. Other recorders are undoubtedly as well suited, but for explicitness, the details of the modification of the Brown recorder will be given.

The last six gears of the chart drive train were removed from their supporting shafts, the gear shafts and the automatic standardization gears being left in place. An adapter, figure 3 , was made to mount a 17 -tooth ratchet gear, 1, drive pawl, 2, and lever, 3 , upon the shaft, 4, of the driving gear of the chart drum. The solenoid magnet, 5, and armature, 6, which operates the rachet lever is mounted upon a $1 / 8$-in. aluminum plate, 7 , which in turn is screwed to the recorder chassis, using existing tapped holes in the chassis. A hold pawl, 8, has been mounted upon the aluminum plate to prevent the chart drum from being pulled back by the tension of the unwinding paper.

It is planned to replace the 17 -tooth gear with a 48-tooth rachet and equip the solenoid with an adjustable stop. In this way the paper can be advanced in steps of multiples of 0.025 in., according to the number of ratchet teeth taken per step.

It is further planned to add a time-marking pen to the recorder so that the chart record will be complete, including temperature, fringe count, and time 


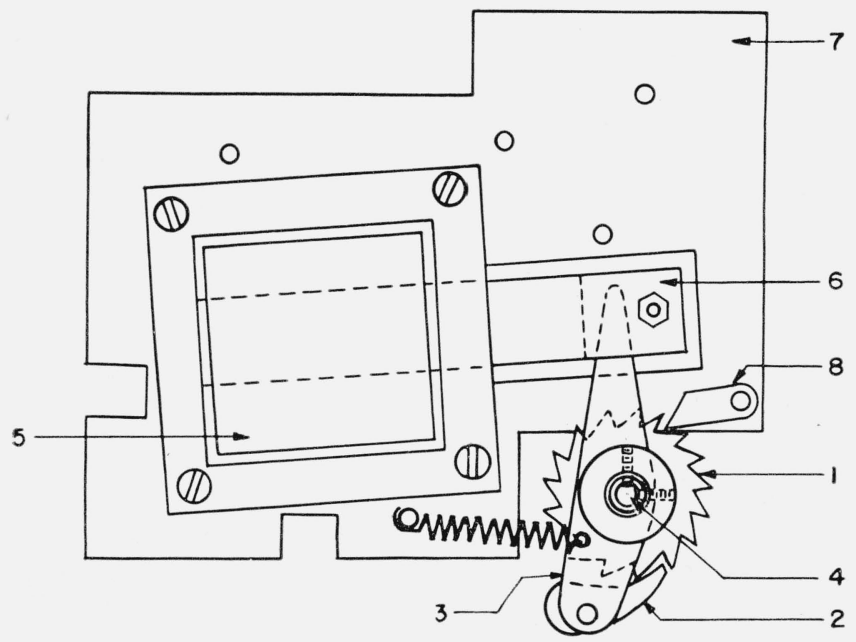

Figure 3. Chart advancing mechanism.

See text for explanation of numbered items.

\section{Procedure}

The sample of rubber to be tested can be cut from a molded sheet of rubber if it is of uniform thickness, or a slice may be cut with a razor from bulk material in an arrangement like a miter box. The faces of the slice should be nearly parallel. Three specimens about $4 \mathrm{~mm}$ square are then cut from the slice or molded sheet. The edges of the test specimens are trimmed to remove any feather-like protrusions that may be present.

The specimens are placed in the wells above the adjusting wedges in the specimen holder. The top interferometer plate is then placed upon the samples and with the eyepiece of the viewing instrument removed, the image reflected from the bottom of the top plate is brought into coincidence with the image from the top of the bottom plate by adjusting the positions of the quartz wedges. The eyepiece is inserted, and the fringe pattern, which is now visible, is adjusted until not more than 10 fringes are visible.

The openings on the side of the specimen holder are closed, and the Dewar is supported in place around the specimen holder. The tripod screws are adjusted to center the return image of the source upon the lower half of the aperture of the iris diaphragm.

Air dried over calcium chloride and dehydrated calcium sulfate is then passed into the chamber in a continuous stream at a pressure about $2 \mathrm{~mm}$ of mercury above atmospheric pressure. Ice is placed in the reference junction bath, and the temperature recording potentiometer is standardized. Liquid nitrogen is then slowly introduced into the Dewar. With a little practice a cooling rate between $1 / 2$ and $1 \mathrm{deg}$ $\mathrm{C} / \mathrm{min}$ can be maintained.

It is necessary to adjust the leveling screws on the viewing instrument occasionally to insure that the image of the light source coincides with the lower half of the aperture of the iris diaphragm. This image moves slightly because of differential contraction of various parts of the specimen holder. The errors due to the image shifts introduced by the adjusting procedure are negligible compared to other errors in the procedure when measurements are made on rubber or other materials of high expansivity.

During the course of a run it will be noted that the number and orientation of the fringes gradually change. These changes are due to the tilting of the top plate relative to the bottom plate. The origin of the tilting is discussed in detail by Saunders [2], so only a brief account need be given here. First, since the samples are not necessarily equal in thickness and wedges are used to make up the difference, the magnitude of change in length of the three specimens will be different tor a given change in temperature. It has already been noted that the error due to this cause is small, but it can be further reduced by placing the aperture in the viewing mirror near the image of one of the specimens and using the thickness of that specimen in the calculation of the coefficient of expansion.

Second, if sticking occurs between the samples and the interferometer plates, the specimens must tilt because of the difference between the expansions of the specimens and the interferometer plate upon a change in temperature. However, if one of the specimens is weighted more heavily than the others, the tilting will take place at the unweighted specimens. Therefore, by placing a small weight (about $1 \mathrm{~g}$ ) upon the top plate above one of the specimens, and placing the aperture of the viewing mirror near that specimen, the tilting error can be reduced. Best practice is to locate the thickest specimen by noting the positions of the wedges and to place the weight above that specimen, since it will usually be found that the weight reduces the height of that specimen slightly. The effect of sticking of the specimens can further be reduced by coating them with graphite, as mentioned by Wood [3].

Occasionally, when going through a transition or when the specimens stick to the interferometer plates, it is found that the number of fringes becomes too great for the phototube to resolve the passage of a single fringe. This difficulty can be readily corrected by an appropriate adjustment of the quartz wedges. It should be noted, however, that such adjustments of the fringe pattern can be serious sources of error. Saunders [2] presents a thorough discussion of the ercors introduced by tilting of the interferometer plate.

The effect of the change in dimensions of the quartz wedges can be taken into account, but since the coefficient of expansion of quartz is about onethousandth that of rubber, no correction is considered necessary.

\section{Treatment of Data}

The second-order transition temperature or other transition temperatures can be obtained directly from the strip-chart record of fringe count versus thermocouple emf. The second-order transition temperature may be defined as the temperature at the intersection of the extrapolated straight portions 


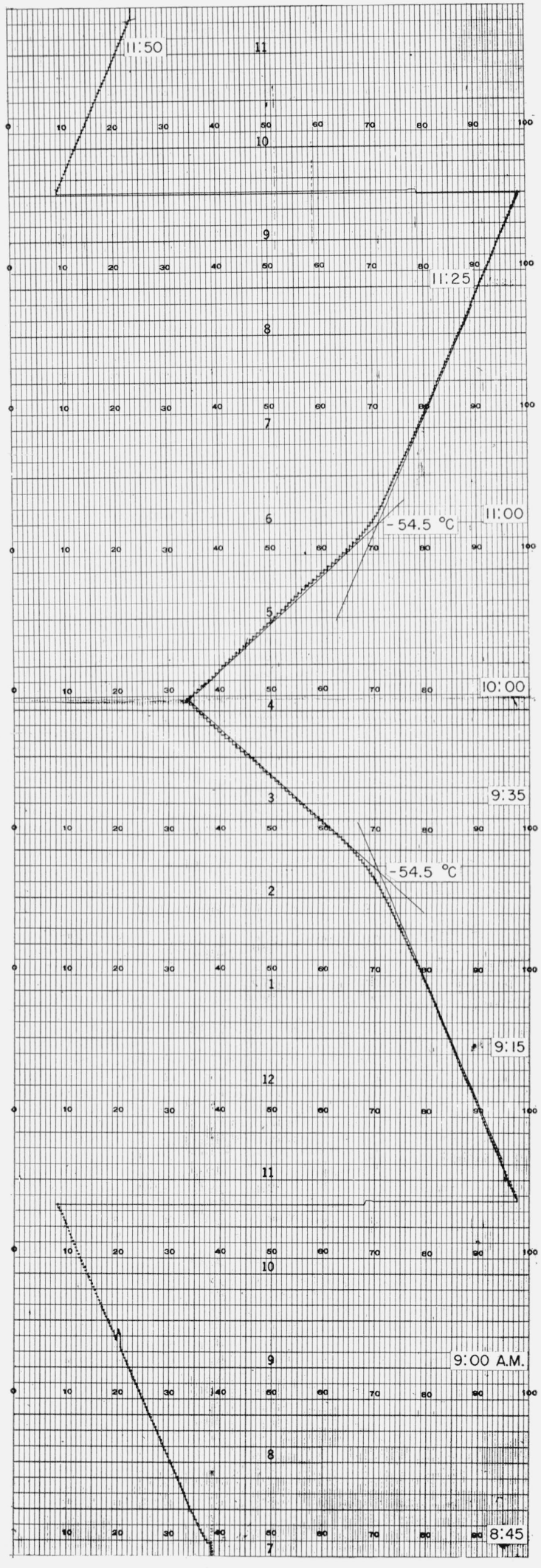

FIGURE 4. Strip chart record, sodium-potassium catalyzed polybutadiene. of the record above and below the transition. The value of the emf corresponding to this point and the corresponding temperature may then be read directly from the chart with the aid of a calibrated scale.

A typical experimental strip-chart record showing the second-order transition at $-54.5^{\circ} \mathrm{C}$ is given in fig. 4. The material tested is a polybutadiene polymerized at $5^{\circ} \mathrm{C}$ with a mixture of sodium and potassium as catalyst in a diluent composed of 150 parts pentane and 50 parts methylcyclohexane. The specimen thickness was $2.90 \mathrm{~mm}$. The illumination was the yellow helium line, 5876 A. The top interferometer plate, $2 \mathrm{~cm}$ in diameter weighed $1.58 \mathrm{~g}$. The second-order transition temperature has been indicated. Time has also been recorded at intervals during the run to indicate the rate of cooling or heating.

The data have been replotted as shown in fig. 5 . The temperature has been obtained from the strip-chart by means of the calibrated scale for every tenth fringe in the linear portions and for every other fringe in the curved regions. The change in length in microns per centimeter, as computed by the method shown below, was then plotted against temperature. It is necessary that the data be treated in this manner if values of the coefficient of expansion are desired, as this method of calculation corrects for the nonlinearity of the temperature-emf curve of the thermocouples and for the change in wavelength of light as the index of refraction of the air in the interferometer changes with temperature.

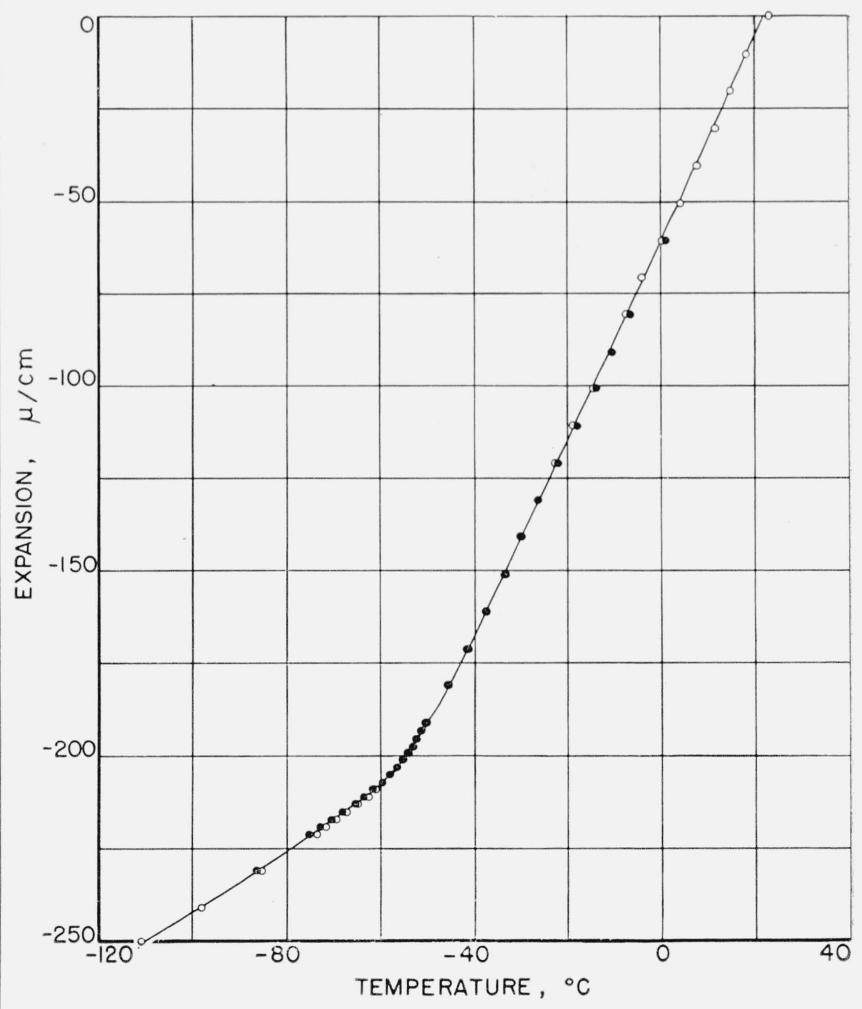

Figure 5. Dimensional changes in sodium-potassium catalyzed polybutadiene.

- Increasing temperature; $\bigcirc$, decreasing temperature. 
The expansion in microns per centimeter is calculated according to the following expression

$$
\frac{\Delta L}{L_{0}}=\frac{\Delta N \lambda}{2 L_{0}}+C
$$

where $\Delta L$ is the expansion of the sample over the temperature range $T_{0}$ to $T ; \Delta N$ is the number of fringes passing the reference point, counting from $T_{0}$, between $T_{0}$ and $T ; \lambda$ is the wavelength of the light used in air at room temperature; $L_{0}$ is the length of the sample at $T_{0}$; and $C$ is the correction for change in wavelength with temperature obtained from a table prepared by Merritt [1].

Experience shows the location of transition temperatures to be reproducible to $\pm 0.5 \mathrm{deg} \mathrm{C}$, where the transition itself is sharp enough to be defined with equal or better precision. Measurements of expansivities of rubber show deviations of about \pm 5 percent.

\section{Other Applications}

In the case of rubber-like materials of a given sample thickness, say $0.3 \mathrm{~cm}$, the passage of 1 to 2 fringes corresponds to a $1 \mathrm{deg} \mathrm{C}$ change in temperature above the transition temperature while below the transition temperature, about half as many fringes correspond to the same temperature interval. Metals, however, have a coefficient about one-tenth and glasses about one twenty-fifth that of rubber. In tests on metals, the number of fringes passing the reference mark is reduced by the same factor for samples of the same thickness, and the number of experimental points along an expansion curve is correspondingly reduced. If the expansion curve is linear or only slightly curved, this is no disadvantage; however, in regions of high curvature it is desirable to have a sufficient number of points to define the curve with reasonable accuracy. The number of experimental points can be increased by increasing the thickness of the sample, but there is a limit to the thickness that can be used witbout too great a loss in fringe contrast unless a differential arrangement [8] is used. It is possible to increase the number of experimental points by arranging a number of switches on the time-intensity recorder spaced to count fractional fringes. It would be necessary before introducing this modification to provide additional stabilization of the power supply to prevent errors due to drift of the output indicator.

The measurement of creep under constant load and the measurement of Young's modulus under light compressive loads suggest themselves as additional applications of the photoelectric recording device.

\section{Conclusions}

The photoelectric fringe counting method can be used to advantage in survey work where it is desired to locate transitions in a large number of materials both rapidly and conveniently. The data can be processed with a minimum of effort and a precision of $\pm 0.5 \mathrm{deg} \mathrm{C}$ or better in the location of transition points can be realized. Values of coefficient of expansion in the case of rubber-like materials are reproducible to at least \pm 5 percent.

\section{References}

[1] G. E. Merritt, An interference method of measuring thermal expansion, J. Research NBS 10, 59 (1933) RP515.

[2] J. B. Saunders, Improved interferometric procedure with application to expansion measurements, J. Research NBS 23, 179 (1939) RP1227.

[3] L. A. Wood, N. Bekkedahl, and C. G. Peters, Application of the interferometer to the measurement of dimensional changes in rubber, J. Research NBS 23, 571 (1939) RP1253.

[4] J. B. Saunders, An instrument for photographing interference phenomena, J. Research NBS 35, 157 (1945) RP1668.

[5] F. C. Nix and D. MacNair, Interferometric dilatometer with photographic recording, Rev. Sci. Instr. 12, 66 (1941).

[6] A. Trobridge, Photograph of moving interference fringes, J. Opt. Soc. Am. 6, 195 (1922).

[7] C. Pulfrich, The Abbe-Fizeau dilatometer, Z. Instrumentenk. 18, 261 (1898).

[8] J. B. Saunders, Expansivity of Vyeor brand glass, J. Research NBS 28, 51 (1942) R P1445.

Washington, December 14, 1950. 